\title{
Spaßgeneration vs. Silver Talents - mit nachhaltigem Personalmanagement dem demografischen Wandel begegnen
}

\author{
Julia Hornung
}

(C) Springer-Verlag Wien 2012

Bei Thema Ressourcenmangel begegnen uns annähernd täglich Rohstoffe wie Wasser, Erdöl \& Co., die für die Produktion verschiedenster Güter unverzichtbar sind. Doch wie gehen wir mit der Verknappung unserer wichtigsten Ressource in der Pflege um, unseren Mitarbeitern?

Nicht erst seit der Diskussion um erneuerbare Energien in der Umweltpolitik steht der res-sourcenschonende und nachhaltige Umgang mit Rohstoffen aller Art im öffentlichen Fokus. Der Grundgedanke der Nachhaltigkeit im Zusammenhang mit ressourcenschonendem Wirtschaften ist nicht neu, stammt er doch ursprünglich aus der Forstwirtschaft und wird als die Nutzung regenerierbarer lebender Ressourcen lediglich in dem Maße wie Bestände natürlich nachwachsen verstanden.

Ähnliches lässt sich auch für das Personalmanagement in der Pflege aussagen, da auch hier Fluktuation auf der einen Seite und Personalakquise auf der anderen Seite den Alltag von Leitungskräften prägen. Es gilt somit, ein nachhaltiges Personalmanagement anzustreben, in dem die Nutzung von Personalressourcen, deren natürlicher alters- oder fluktuationsbedingter Abbau sowie die langfristige Sicherstellung von neuen Ressourcen in Balance sind.

Stein des Anstoßes in der Diskussion um Fach- und Führungskräfte bildet häufig der demografische Wandel. Die klassische Bevölkerungspyramide in Form eines Tannenbaums verändert sich bereits seit einigen Jahren und aus der Tanne wird immer mehr ein Laubbaum, mit einer großen Krone aus einer Vielzahl an älteren Menschen in unserer Bevölkerung und einem schmalen, aber noch stabilen Stamm aus jungen Menschen, deren Aufgabe es ist die Baumkrone - nicht nur symbolisch - zu tragen.

Um den Auswirkungen des demografischen Wandels strategisch zu begegnen und damit auch langfristig über die notwendigen Personalressourcen in Quantität und Qualität zu verfügen, bedarf es eines nachhaltigen Personalmanagements, welches die Pflege und ihre Akteure unterstützt und fördert. Ziel muss es hierbei sein, nicht nur altersfokussiert zu agieren, sondern generationenorientiert und bedarfsgerecht Maßnahmen anzustoßen, um Alt und Jung gerecht zu werden.

Mit seinen 5 Bausteinen bildet das 5-Säulen-Konzept einen Ansatz, dem demografischen Wandel durch nachhaltiges Personalmanagement zu begegnen. Die 5 modularen Säulen, welche es einrichtungsindividuell umzusetzen gilt sind:

- Gesundheitsmanagement

- Lebenslanges Lernen

- Organisation und Arbeitsgestaltung

- Personal- und Rekrutierungspolitik

- Führung

J. Hornung, MA $(\triangle)$

Sozialwerk St. Georg, Gelsenkirchen, Deutschland 